\title{
Investigating Gender Effect on Traditional and Herbal Remedies to Manage Diabetes in KSA
}

\author{
Yahya M. AlMurtadha, Osman A. Abdalla, Abdelrahman O. Elfaki, and Mustafa H. Alfrraj
}

\begin{abstract}
Consulting Traditional healers and use herbs for managing diabetes are common in Saudi Arabia despite the major development in health care systems. This study aims at investigating the gender effect on managing diabetes for those consult traditional healers and those use herbs to raise the alarm for the medical policy makers of the reasonable percentage of patients following such traditional remedies. The research used the Saudi Arabia NCD2005 obtained from WHO site the data reported as a result of the national survey of non-communicable disease in 2005 . The investigation will start with statistical analysis followed by a predictive analysis of gender factor for those managing diabetes by consulting traditional healers or using herbs using regression analysis (support vector machine) as a data mining technique. Rapidminer was used as a data mining software for predicting which gender group (Men or Women) most attracted to those kinds of traditional remedies. The findings show that gender is a significant factor helps to predict the traditional remedy type. The results highlight that women are more affected by consulting traditional healers than men while men are better in managing diabetes by using herbs. Though, it is recommended for the medical policy makers to encourage the medical doctors to discuss with their patients if they are using such traditional remedies which may affect the medical therapy. Future work includes comparing the effectiveness of managing diabetes in Saudi Arabia by modern health care medication against using herbs.
\end{abstract}

Index Terms - Data mining, diabetes traditional healers, diabetes herbs remedy, gender and diabetes, diabetes in KSA.

\section{INTRODUCTION}

The Gulf Cooperation Council (GCC) countries increase in wealth because of the energy boom in the past decade, which has brought with it radical changes in lifestyle that have led to a significant increase in chronic diseases such as the increasing number of people diagnosed with diabetes [1]. With the economic growth, people tend to move to urban centers and found likely to adapt lifestyle that contribute to increasing high-calorie food consumption and sedentary lifestyle [2]. The citizens of GCC have changed their nutrition from "predominantly consuming dates, milk, fresh vegetables and fruit, whole wheat bread and fish mainly foods rich in

Manuscript received September 11, 2014; revised November 13, 2014 This work was supported by the Deanship of Scintific research, University of Tabuk under Grant S-1435-0132.

Yahya M. AlMurtadha and Abdelrahman O. Elfaki are with the Department of Computer Science, Faculty of Computers and Information Technology, University of Tabuk, Tabuk, Kingdom of Saudi Arabia (e-mail: y.murtadha@ut.edu.sa, a.elfaki@ut.edu.sa).

Osman A. abdalla is with the Department of Information Technology, Faculty of Computers and Information Technology, University of Tabuk. Kingdom of Saudi Arabia (e-mail: osman_uofg@ut.edu.sa).

Mustafa H. Alfrraj is with the Diabetes Center, King Khalid Hospital, Tabuk, Kingdom of Saudi Arabia (e-mail: dr_mh2009@ @otmail.com). saturated fat and high refined diets combined with low dietary fiber" [2]. Fig. 1 [3] shows the expected increasing in the number of people diagnosed with diabetes globally.

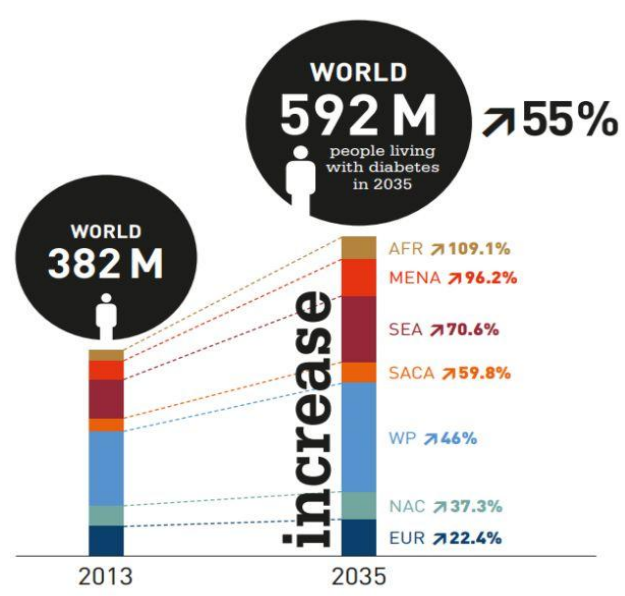

Fig. 1. Expected increasing in the number of people diagnosed with diabetes (Source: International-Diabetes-Federation).

According to International Diabetes Federation [4] 1 in 9 adults in this Middle East and North Africa region has diabetes and More than half of people with diabetes in this region don't know they have it and reported Saudi Arabia among the 10 countries with the highest diabetes prevalence rates in the world. In 2002, the WHO warned that obesity was an important determinant of health that could lead to increased levels of resistance to insulin and in turn raise the risk of type 2 diabetes mellitus [5]. Overweight and obesity as signs of possible diabetes are high in Saudi Arabia especially among 30-60 years with a great sign of prevalence among preschoolers leading to very high rate of diabetes level raising the alarm for national survey to investigate the importance of various factors that contribute to these troubling trends [6]. According to the World health Organization, around 22\% (males) and 21.7\% (females) of adults aged 25 and over in KSA had raised blood glucose in 2008 [7].

Recent research in Saudi Arabia shows that the number of patients with Diabetes Mellitus is increasing drastically [8]. Ministry of Health, Saudi Arabia in cooperation with World Health Organization reported the NCD 2005 report for the survey conducted to scan the national standard Non-Communicable Disease risk factor. NCD 2005 represents a national situational analysis and a baseline survey of NCD and their risk factors conducted in 2005. A previous study has reported the diabetes major risk factors as a shift towards more Western diets, Lower levels of physical activity and Genetic ability for diabetes [1].

The objective of this study is to investigate gender factor affecting choosing herbal medication and/or consulting 
traditional healer in Saudi Arabia to guide the medical researches on gender importance to overcome a lack of knowledge among doctors about herbal medicine.

\section{RELATED WORKS}

Although GCC countries like Saudi Arabia and Oman witnessed major development in the health care system, wide range of people still prefer to use traditional and herbs before visiting the clinics. This medication is prescribed by traditional healers who pass this knowledge from one generation to another and is regarded as a heritage [9], [10]. In addition, such kind of remedy is used for several resource-limited territories/countries suffering from the challenge of diabetes management since those healers can easily understand and collaborate to transfer the prevention strategies to the patients [11].

Herbal treatment: is a common method of fighting diseases globally alongside conventional drug therapy. Approximately $75-80 \%$ of the world populations consider herbal medication particularly widespread in the developing countries for many reasons [12]. Those reasons include culturally acceptance like Chinese [13], Sudan [14], Saudi Arabia [15] and many other countries, less dangerous and a more natural form of medicine that is compatible with the human body [15]. Furthermore as a study in Sudan reported that the proposed motivations for using herbal medicines were easy to get, low cost and positive recommendation by others [14].

A study in Saudi Arabia found that $91.1 \%$ of the participants did not consult or take advice from a physician prior to purchasing herbal remedies for concurrent use alongside conventional medication [15]. Several works reported the side effects, and sequences of using herbal medicine without proper, safe and specialist investigation. Al-Faris [16] conducted a survey in Riyadh, Saudi Arabia and concluded that There is a need to educate the public through the media and health professionals on the appropriate use of alternative medicine. As a remarkable conclusion by [17] that there is a lack of understanding in Middle East and Africa of diabetes and its treatments by both physicians and patients requires more and better diabetes education. Ali and his colleagues [14] proposed to integrate herbal medicine into the current medical curriculum so that in future physicians will be better able to communicate with their patients.

A study in Saudi Arabia found that there is a good understanding toward integrating herbal medicine into primary care services among a group of patients attending a primary care center in Saudi Arabia [18]. Otoom et al. [19] reported the use of medicinal herbs among diabetic patient in Jordan is common and concluded to investigate the efficiency and safety of using such medication. Use of herbal therapy also found to be a common diabetes medication in Turkey with a recommendation to be educated by the health professionals [20]. Several studies reported the efficiency and safety of using herbs in medication. A Chinese study reported that Treatment with a Tianqi capsule for 12 months significantly decreased the incidence of type 2 diabetes, and this herbal drug was safe to use [21]. A study in Saudi Arabia concluded that Nigella sativa is a safe herbal product [22]. One main problem of herbal medicine is the fear of patients to talk regarding use of herbs with doctors as it may affect the outcome and the management of their disease [23].

\section{MATERIALS AND METHODS}

\section{A. Data Set}

Ministry of health of Saudi Arabia in cooperation with WHO carried out a national survey of Non-Communicable Diseases NCD risk factors including diabetes through interview, physical examination and laboratory examination of blood samples of study participants; based upon the WHO STEPWise@ approach. This study will use the part of healing diabetes by consulting a traditional healer or by herbal treatment reported in the NCD2005 report.

\section{B. Methodology}

Data in the raw format is not ready for various statistical and data mining techniques. Transformation to the suitable format for the purpose of mining and analysis is necessary. During this step, the data of NCD 2005 pertaining traditional and herbs remedies will be transformed to the format accepted by the required analysis technique. STATISTICA software will be used to analyze the gender effect on managing the diabetes. Following that is a regression analysis based on the Support Vector Machine technique to investigate which method of treatment method seems to show improvement in managing diabetes: consulting traditional healers or taking herbs. Full details of the statistical and data mining analysis in the following subsections. Finally, a conclusion and remarks of the results will be given. Fig. 2 gives an overview of the data preparation and analysis.

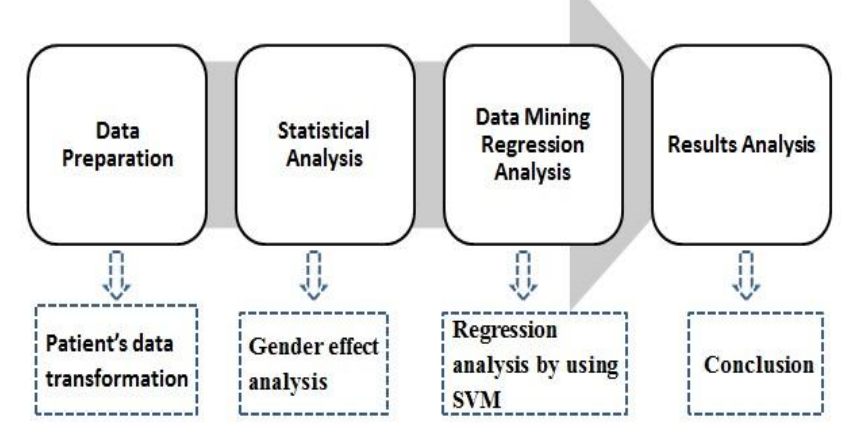

Fig. 2. Analysis steps.

\section{Statistical ANALYSIS}

Standard statistical software (STATISTICA) and MS Excel were used for the data management and statistical analysis. The results were considered statistically significant when $p \leq 0.05$. The final analysis included 2 tables, Table I for those consulted traditional healers and the Table II is for those who used herbs for diabetes medication. Both tables include data for "men" and "women". The data included 619 diabetes patients in Saudi Arabia at the time of the survey in 2005. 355 $(51.3 \%)$ of them are men, and $336(48.6 \%)$ are women. The data is separated to five age categories: $15-24,25-34,35-44$, 45-54 and 55-64 years old. For each gender and age group, 
the data contained two values: "Big-N" indicates the total number of diabetes patients of the corresponding age and gender group and "small-n" indicates the number of patients show improvement in controlling the diabetes by following the corresponding healing type indicated by the table name. For example: for the "gender=men "and "age group= 55-64"; 6 patients (percentage $=4.5 \%, \mathrm{SE}=2.00 \%$ ) out of 142 patients show improvement in controlling diabetes by consulting traditional healers while 5 patients (percentage $=3.5 \%$, $\mathrm{SE}=1.9 \%$ ) show improvement by following herbal treatment. Considering women group, for the "gender=women "and "age group $=55-64 " ; 9$ patients (percentage $=9.9 \%, \mathrm{SE}=3.3 \%$ ) out of 89 patients show improvement in controlling diabetes by consulting traditional healers while 6 patients (percentage $=6.7 \%, \quad \mathrm{SE}=2.7 \%$ ) show improvement by following herbal treatment.

Normally, t-test is used to calculate the significant differences between the two groups of sample. In this study the hypotheses that we are testing are:

H0: Gender shows no difference in consulting traditional healers or using herbs to manage diabetes.

H1: Gender is a significant factor affecting consulting traditional healers or using herbs to manage diabetes.

TABLE I: CONSULTATION WITH TRADITIONAL HEALERS BY THOSE WITH DIABETES: MEN AND WOMEN

\begin{tabular}{|c|c|c|c|c|}
\hline Age & $\mathbf{N}$ & $\mathbf{n}$ & SE & gender \\
\hline $15-24$ & 11 & - & - & 1 \\
\hline $25-34$ & 13 & 3 & $\begin{array}{l}13.80 \\
\%\end{array}$ & 1 \\
\hline $35-44$ & 70 & 6 & $4.00 \%$ & 1 \\
\hline $45-54$ & $\begin{array}{l}13 \\
0\end{array}$ & 9 & $2.30 \%$ & 1 \\
\hline 55-64 & $\begin{array}{l}14 \\
2\end{array}$ & 6 & $2.00 \%$ & 1 \\
\hline $15-24$ & 12 & 2 & $\begin{array}{l}10.30 \\
\%\end{array}$ & 2 \\
\hline $25-34$ & 28 & 3 & $5.60 \%$ & 2 \\
\hline $35-44$ & 94 & 6 & $2.50 \%$ & 2 \\
\hline $45-54$ & $\begin{array}{l}12 \\
4\end{array}$ & $\begin{array}{l}1 \\
6\end{array}$ & $3.20 \%$ & 2 \\
\hline 55-64 & 89 & 9 & $3.30 \%$ & 2 \\
\hline
\end{tabular}

TABLE II: USING HERBALS BY THOSE WITH DiABETES: MEN AND WOMEN

\begin{tabular}{lllll}
\hline \hline Age & $\mathrm{N}$ & $\mathrm{n}$ & $\mathrm{SE}$ & gender \\
\hline 1 & 11 & - & - & 1 \\
2 & 13 & - & - & 1 \\
3 & 70 & 5 & $3.40 \%$ & 1 \\
4 & 130 & 7 & $2.10 \%$ & 1 \\
5 & 142 & 5 & $1.90 \%$ & 1 \\
1 & 12 & 2 & $10.30 \%$ & 2 \\
2 & 28 & 2 & $4.50 \%$ & 2 \\
3 & 94 & 4 & $2.30 \%$ & 2 \\
4 & 124 & 10 & $2.50 \%$ & 2 \\
5 & 89 & 6 & $2.70 \%$ & 2 \\
\hline \hline
\end{tabular}

Prior to apply t-test, the original data must be in a specific form suitable for computing. Table I and Table II show the formatted data after preparing for STATISTICA whereby the gender factor has been coded to 1="Men" and 2="Women", $\mathrm{N}$ represents the total population of patients with diabetes and $\mathrm{n}$ represents the population of diabetes patients show improvement in managing diabetes. When applying t-test using STATISTICA, we set the Gender as the grouping variable and $n$ as the dependent variable. Table I is for those consult traditional healers and Table II for those using herbals for managing diabetes.

Applying t-test on the data of healing by consulting traditional healers, a significant differences were found between the number of patients show improvement in controlling the diabetes "small- $\mathrm{n}$ " and the gender ( $p$ value $=$ 0.006952). this result is similar to a previous study by [14]. Age group was also found to be a significant factor when associated with the gender $(p$ value $=0.007685)$. Using t-test for the data of healing by taking herbs; also supported the significance of gender in show improvement ( $p$ value $=$ 0.009167). It was found also that there is a strong association between the age group and the number of patients show improvement $(p$ value $=0.041156)$. Based on the t-test results and $p$ values, we conclude that hypothesis $\mathrm{H} 1$ is true which indicates that gender is a significant factor in following the diabetes traditional remedies. Further investigation is needed to determine what gender group (men or women) is more attracted to what diabetes traditional remedy. This is the task to be accomplished by applying the prediction analysis using data mining as will be explained the following section.

\section{DATA Mining REgRESSION ANALYSIS}

Regression analysis is used to investigate the relationship(s) may exist between dependent variables and independent variables then report the effect of varying each independent variable's values on the dependent variable while the other independent variables are fixed. In data mining, regression analysis is well known for "prediction" or "forecasting" to find the most effective independent variable(s). Rapid miner tool provides a variety of regression analysis such as linear regression, logistic regression and support vector machine (SVM) which are the most common methods for regression analysis in data mining. Recall as an evaluation metric is the sum of true prediction divided by the number of cases that belong to the actual class. Using SVM technique on the data of those consult a traditional healers, Table III shows the confusion matrix describing the prediction accuracy of gender group for those consult traditional healers to control diabetes. The class recall of women group is $100 \%$ compared to the class recall of the men group which is only $40 \%$. The matrix indicates that the "women" group is more predictable to accept the traditional healers remedy than the "men" group" with prediction accuracy of $70 \%$.

TABLE III: SVM CONFUSION MATRIX FOR THOSE CONSULT TRADITIONAL HEALER

\begin{tabular}{lll}
\hline \hline Accuracy : 70\% & & \\
\hline & Predicted $\mathrm{m}$ & Predicted $\mathrm{w}$ \\
\hline Predicted $\mathrm{m}$ & 2 & 0 \\
& & 5 \\
Predicted $\mathrm{w}$ & 3 & $100 \%$ \\
Class recall & $40 \%$ & \\
\hline \hline
\end{tabular}


Table IV illustrates the confusion matrix describing the prediction accuracy of gender group for those using herbs to manage diabetes. Table IV states that men group shows better management when use herbs for medicine than women group with prediction accuracy of $70 \%$. The class recall of men group is $100 \%$ compared to the class recall of the women group which is only $40 \%$.

TABLE IV: SVM CONFUSION MATRIX FOR HERBAL TREATMENT

\begin{tabular}{lll}
\hline \hline Accuracy : 70\% & & \\
\hline & Predicted $\mathrm{m}$ & Predicted $\mathrm{w}$ \\
\hline Predicted $\mathrm{m}$ & 5 & 3 \\
Predicted $\mathrm{w}$ & 0 & 2 \\
Class recall & $100 \%$ & $40 \%$ \\
\hline \hline
\end{tabular}

\section{DISCUSSION AND CONCLUSION}

Diabetes is increasing in KSA. Herbal medication is like a heritage in many countries especially KSA. In addition to medical therapy several diabetes remedies are considered including consulting traditional healers and using herbs to manage diabetes. This study investigated the effect of gender in choosing either consulting traditional healers or using herbs The results have shown that gender is an effective factor. Data mining regression analysis shows that women are more accepted to consult traditional healers than men. In the other hand, men are more accepted to use herbs to manage diabetes than women. Using these foundlings, medical policy makers may include such topics in the medical schools to encourage diabetes patients to talks about using traditional remedies since Men and women normally denied talking about traditional remedies with their doctors causing potential conflict with the medication therapy. Future works may consider study the demographic factor to locate the areas in KSA use herbs to manage diabetes and investigating the effect of such diabetes management therapies.

\section{ACKNOWLEDGEMENT}

The authors would like to acknowledge financial support for this work, from the Deanship of Scientific Research (DSR), University of Tabuk, Tabuk, Saudi Arabia, under grant no. S-1435-0132.

\section{REFERENCES}

[1] L. Klautzer, J. Becker, and S. Mattke, "The curse of wealth-Middle Eastern countries need to address the rapidly rising burden of diabetes," International Journal of Health Policy and Management, vol. 2, no. 3, p. 109, 2014.

[2] M. Badran and I. Laher, "Type II diabetes mellitus in Arabic-speaking countries," International Journal of Endocrinology, vol. 2012, pp. 2012.

[3] IDF. (2014). Diabetes: facts and figures. [Online]. Available: http://www.idf.org/worlddiabetesday/toolkit/gp/facts-figures

[4] IDF. (2012). Diabetes Atlas. 5th edition. [Online]. Available: http://www.idf.org/sites/default/files/5E_IDFAtlasPoster_2012_EN.p df

[5] J. Guilbert, The World Health report 2002-Reducing Risks, Promoting Healthy Life, Education for Health-Abingdon-Carfax Publishing Limited, vol. 16, no. 2, pp. 230-230, 2003.

[6] S. W. Ng et al., "The prevalence and trends of overweight, obesity and nutrition-related non-communicable diseases in the Arabian Gulf States," Obesity Reviews, vol. 12, no. 1, pp. 1-13, 2011.

[7] World-Health-Organization. (2013). Global Health Observatory (GHO). [Online]. Available: http://www.who.int/gho/en/
[8] A. A. Aljumah, M. G. Ahamad, and M. K. Siddiqui, "Application of data mining: Diabetes health care in young and old patients," Journal of King Saud University- Computer and Information Sciences, vol. 25, pp. 127-136, 2013

[9] G. E. El-Ghazali et al., "Traditional medicinal plants indigenous to Al-Rass province, Saudi Arabia," Journal of Medical Plants Res., vol. 4, no. 24, pp. 2680-2683, 2010.

[10] R. M. Al-Kindi, et al., "Complementary and alternative medicine use among adults with diabetes in Muscat region, Oman," Sultan Qaboos University Medical Journal, vol. 11, no. 1, pp. 62, 2011.

[11] G. N. Mbeh et al., "Traditional healers and diabetes: results from a pilot project to train traditional healers to provide health education and appropriate health care practices for diabetes patients in Cameroon," Global Health Promotion, vol. 17, no. 2, pp. 17-26, 2010.

[12] C. Clair et al., "Association of smoking cessation and weight change with cardiovascular disease among adults with and without diabetes," Journal of American Medical Association, vol. 309, no. 10, pp. 1014-1021, 2013.

[13] S. Jiang et al., "Comparative metabolites in plasma and urine of normal and type 2 diabetic rats after oral administration of the traditional Chinese scutellaria-coptis herb couple by ultra performance liquid chromatography-tandem mass spectrometry," Journal of Chromatography B, vol. 965, pp. 27-32, 2014.

[14] B. A. M. Ali and M. S. Mahfouz, "Herbal medicine use among patients with type 2 diabetes in North Sudan," Annual Research \& Review in Biology, vol. 4, no. 11, pp. 1827-1838, 2014.

[15] A. Suleiman, "Attitudes and beliefs of consumers of herbal medicines in Riyadh, Saudi Arabia," Journal of Community Med Health Educ, vol. 4, no. 269, pp. 2161-0711, 2014

[16] E. A. Al-Faris, "The pattern of alternative medicine use among patients attending health centres in a military community in Riyadh," Journal of Family \& Community Medicine, vol. 7, no. 2, pp. 17, 2000.

[17] M. Almaatouq et al., "Barriers to the delivery of optimal antidiabetic therapy in the Middle East and Africa," International Journal of Clinical Practice, vol. 68, no. 4, pp. 503-511, 2014.

[18] S. Allam, M. Moharam, and G. Alarfaj, "Assessing patients' preference for integrating herbal medicine within primary care services in Saudi Arabia," Journal of Evidence-Based Complementary \& Alternative Medicine, vol. 19, no. 3, pp. 205-210, 2014.

[19] S. Otoom et al., "The use of medicinal herbs by diabetic Jordanian patients," Journal of Herbal Pharmacotherapy, vol. 6, no. 2, pp. 31-41, 2006.

[20] N. Inanç et al., "Use of herbs by the patients with diabetes in Kayseri, Turkey," Pakistan Journal of Nutrition, vol. 6, no. 4, pp. 310-312, 2007.

[21] F. Lian et al., "Chinese herbal medicine tianqi reduces progression from impaired glucose tolerance to diabetes: A double-blind, randomized, placebo-controlled, multicenter trial," The Journal of Clinical Endocrinology \& Metabolism, vol. 99, no. 2, pp. 648-655, 2014.

[22] A. Bamosa, "Nigella sativa is a safe herbal product," Journal of Integrative Medicine, vol. 12, no. 1, pp. 66-66, 2014.

[23] N. A. Al-Rowais, "Herbal medicine in the treatment of diabetes mellitus," Saudi Medical Journal, vol. 23, no. 11, pp. 1327-1331, 2002.

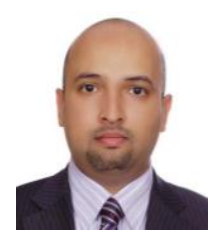

Yahya is an assistant professor at the Computer Science Department, Faculty of Computers and Information Technology, University of Tabuk, KSA since 2012. Before that, he worked in many countries as he was a senior lecturer at Asia Pacific University of Technology and Innovation, Malaysia and a lecturer and department head in Sana'a Community College, Yemen. He participated in many researches and funded projects in the field of Artificial Intelligence and data mining. He published many articles and attended several international conferences in the area of AI, software agents, data mining and biotechnology.

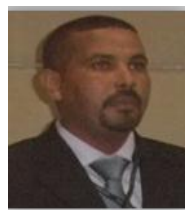

Osman is an assistant professor at the University of Tabuk, Saudi Arabia. Previously, he was an assistant professor at the University of Science and Technology in Sudan. He has participated in many projects and research related to artificial intelligence in Malaysia, Sudan and KSA. The fields of his interests include 
artificial intelligence and data mining. He has published many papers that covered his current areas of interest.

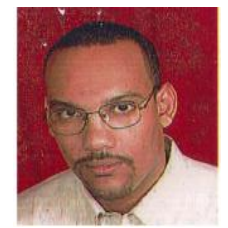

Abdelrahman is an assistant professor at Tabuk University, KSA. Before, He has been a senior lecturer at Management and Science University in Malaysia. He was involved in many projects and researches related to software engineering in different countries which is refined his software engineering experience in both practical and academic fields. His current interests are modeling software product lines engineering and managing variability. He has published many papers related to his current interests.

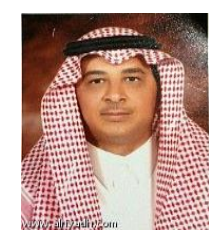

Mustafa Alfrraj is a family medical consultant and the director of Diabetes center, King Khalid hospital, Tabuk, Saudi Arabia. Before that he was the vice general manager of the public health of Tabuk. 